Ribeiro, N.K.R.; Leite, L.L.B.; Pontes, Z.B.V.S. Revista Eletrônica de Farmácia Vol. X (1), $16-26,2013$.

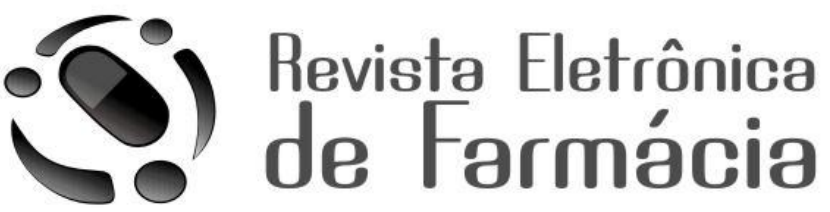

REF - ISSN 1808-0804 Vol. X (1), 16 - 26, 2013.

\title{
ESTUDO FARMACOEPIDEMIOLOGICO: 0 USO DE MEDICAMENTOS POR GESTANTES
}

\author{
PHARMACOEPIDEMIOLOGY STUDY: THE USE OF DRUGS BY \\ PREGNANT WOMEN
}
${ }^{1 *}$ Núbia Kellly Rodrigues Ribeiro, ${ }^{2}$ Lidia Lúcia Bezerra Leite, ${ }^{3}$ Zélia Braz Vieira da Silva Pontes

\footnotetext{
${ }^{1}$ Universidade Federal da Paraíba. Professora do Departamento de Ciências Farmacêuticas da Universidade Federal da Paraíba.

${ }^{2}$ Universidade Federal da Paraíba. Graduanda do curso de Farmácia. ${ }^{3}$ Universidade Federal da Paraíba. Professora Doutora do Departamento de Ciências Farmacêuticas da Universidade Federal da Paraíba.

*autor para correspondência: nubiakribeiro@yahoo.com.br
}

Recebido em 21/06/2012, Aceito em 04/02/2013

\section{RESUMO}

Os estudos de utilização dos medicamentos são capazes de fornecer informações importantes para o planejamento em saúde. Com o objetivo de contribuir com o uso racional de medicamentos em gestantes, o presente trabalho buscou determinar o perfil epidemiológico dos medicamentos utilizados pelas gestantes usuárias do serviço de pré-natal do Hospital Universitário Lauro Wanderley (HULW) em João Pessoa - PB. Foi realizado um estudo transversal, após aprovação em comitê de ética, com uma população de 90 gestantes em idades gestacionais diversas atendidas no HULW, classificando os medicamentos de acordo com a categoria de risco do FDA. Os medicamentos, de acordo com a classificação ATC, mais utilizados pelas gestantes foram: o sulfato ferroso (B03 preparados antianêmicos) 31,03\%; paracetamol (N02 analgésicos) 4,93\%; brometo de 
Ribeiro, N.K.R.; Leite, L.L.B.; Pontes, Z.B.V.S. Revista Eletrônica de Farmácia Vol. X (1), $16-26,2013$.

escopolamina (A03 medicamentos para disfunções funcionais gastrointestinais) 4,93\%; dimenidrinato (A04 antieméticos) 2,46\%; metildopa (C02 antihipertensivos) 1,97\%; dipirona (M01antiinflamatórios) 1,45\%, e nistatina (J02 antimicótico) $0,99 \%$. A percentagem de automedicação na população estudada foi de $5,42 \%$ e cerca de $11 \%$ das entrevistadas afirmaram desconhecer 0 medicamento em uso e/ou sua indicação. Nenhum medicamento descrito enquadrou-se nas categorias $D$ ou $X$ da FDA; no entanto, 75,0\% dos medicamentos utilizados pertencem às classes $B$ e $C$, que não há estudos adequados em humanos que comprovem sua segurança. Os resultados deste estudo convergem para os achados da literatura, reforçando a necessidade de caracterizar-se o perfil de utilização de medicamentos em gestantes para poder promover o uso racional dos medicamentos, considerando os possíveis danos que estes podem causar ao feto.

Palavras-chave: Medicamentos. Farmacoepidemiologia. Gestantes.

\section{ABSTRACT}

Studies on drug use can provide important information for health planning. In view of contributing with the rational use of drugs by pregnant women, the present study aimed to determine the epidemiological profile of drugs used by pregnant women attending the prenatal service at Lauro Wanderley University Hospital (LWUH) in João Pessoa, PB. This was a cross-sectional research, approved by the ethics committee, consisting of a population of 90 pregnant women going through different gestational periods. Drugs were classified according to risk category by FDA. The drugs most used by pregnant women, in accordance with the ATC classification, were: ferrous sulfate (B03 antianemic preparations) - 31.03\%; paracetamol (N02 painkillers) - 4.93\%; scopolamine bromide (A03 drugs for functional gastrointestinal disorders) - 4.93\%; dimenhydrinate (A04 antiemetics) 2.46\%; methyldopa (CO2 anti-hypertensive) - 1.97\%; dipyrone (M01 antiinflammatory) - 1.45\%; and nystatin (J02 antifungal) - $0.99 \%$. The percentage of self-medication in the studied population was $5.42 \%$, and about $11 \%$ of the interviewee reported not knowing the drug in use and/or its indication. No drug described was included into categories $D$ or $X$ of the FDA. On the other hand, $75.0 \%$ of them belong to the classes $B$ and $C$, and there are no studies properly designed for humans which can attest their safety. The outcomes reported herein corroborate with the literature findings, reinforcing the need for outlining the profile of drugs used by pregnant women, in order to promote their rational use, particularly because fetus may suffer damages.

Descriptors: Pharmaceutical Preparations. Pharmacoepidemiology. Pregnant Women. 
Ribeiro, N.K.R.; Leite, L.L.B.; Pontes, Z.B.V.S. Revista Eletrônica de Farmácia Vol. X (1), $16-26,2013$.

\section{RESÚMEN}

Los estudios sobre el consumo de medicamentos pueden proporcionar informaciones importantes para la planificación sanitaria. En vista de contribuir con el uso racional de los medicamentos por mujeres embarazadas, el presente estudio tuvo como objetivo determinar el perfil epidemiológico de los medicamentos utilizados por mujeres embarazadas que acuden a los servicios prenatales en el Hospital Universitario Lauro Wanderley (HULW) en João Pessoa, PB. Se trata de una investigación transversal, aprobada por el comité de ética, que consiste en una población de 90 mujeres embarazadas pasando por diferentes períodos gestacionales. Los medicamentos fueron clasificados de acuerdo a la categoría de riesgo por la FDA. Los fármacos más utilizados por las mujeres embarazadas, de acuerdo con la clasificación ATC, fueron: sulfato ferroso (B03 preparados antianémicos) - 31,03\%; paracetamol (N02 analgésicos) - 4,93\%; bromuro de escopolamina (A03 medicamentos para los trastornos gastrointestinales funcionales) - 4,93\%; dimenhidrinato (A04 antieméticos) - 2,46\%; metildopa (C02 anti-hipertensivo) - 1,97\%; dipirona (M01 anti-inflamatorio) - 1,45\%, y nistatina (J02 antifúngico) - 0,99\%. El porcentaje de automedicación en la población estudiada fue de $5,42 \%$, y $11 \%$ de los entrevistados manifestaran no conocer el fármaco en uso y / o su indicación. Ningún fármaco descrito se incluyó en las categorías D o X de la FDA. Por otro lado, 75,0\% de los medicamentos pertenecían a las clases B y C, y no hay estudios diseñados adecuadamente para los seres humanos que puedan garantizar su seguridad. Los resultados encontrados corroboran con la literatura, lo que refuerza la necesidad de trazar el perfil de las drogas utilizadas por mujeres embarazadas, con el fin de promover su uso racional, sobre todo porque el feto puede sufrir daños.

Descriptores: Preparaciones Farmacéuticas. Farmacoepidemiología. Mujeres Embarazadas.

\section{INTRODUÇÃO}

$\mathrm{Na}$ sociedade moderna, o medicamento representa um importante instrumento terapêutico para o tratamento de diversas doenças, mas também ocupa, indevidamente, o lugar de símbolo de saúde passando a partir do momento em que assume tal papel a imprimir que 0 estado patológico seja considerado um fato essencialmente orgânico, cujo único meio de enfrentamento é a utilização da ferramenta medicamento ${ }^{(1)}$. No entanto, visualizar medicamento 
Ribeiro, N.K.R.; Leite, L.L.B.; Pontes, Z.B.V.S. Revista Eletrônica de Farmácia Vol. X (1), $16-26,2013$.

como símbolo de saúde ultrapassa a barreira cultural e ao adentrar no campo da epidemiologia absorve uma outra função amplamente discutida na última década, a função de medicamento como indicador de saúde.

Deficiências estruturais e funcionais das políticas de saúde levam a um consumo exacerbado e indiscriminado de medicamentos, que muitas vezes são utilizados como medidas saneadoras de problemas básicos. A omissão no que diz respeito ao controle da produção e da comercialização de medicamentos por parte do governo e a intensa atividade promocional da indústria farmacêutica influenciam hábitos de prescrição para a população em geral ${ }^{(2)}$.

Com o aumento da expectativa de vida da população brasileira e consequente envelhecimento da população os estudos de utilização de medicamentos passam a afirmar sua importância enquanto meio de estabelecer o perfil de prescrição e lou consumo deste insumo por uma população onde está havendo um incremento na prevalência de doenças crônicas e logo também no instrumento utilizado como forma de controle dessas doenças.

Observa-se um $\begin{array}{r}\text { número } \\ \text { crescente de }\end{array}$ estudos
farmacoepidemiológicos
envelhecimento no Brasil que
abordam diferentes aspectos, tais

como os fatores associados ao uso de medicamentos, a polifarmácia, a automedicação, o alto custo dos medicamentos, seu uso inadequado, as reações adversas aos medicamentos, as interações medicamentosas, bem como os problemas de complexidade das prescrições $^{(3)}$.

Os estudos de utilização de medicamentos (EUMs) compreende ações que envolvem praticamente todo o ciclo da assistência farmacêutica com enfoque em suas conseqüências sanitárias, sociais e econômicas. Assim sendo, os EUMs disponibilizam informações relevantes sobre prevalência, tendências e uso de medicamentos pela comunidade, constituindo uma ferramenta relevante para o planejamento $\mathrm{e}$ implantação de políticas públicas que visem 0 uso racional de medicamentos.

Grandes estudos na área
perinatal envolvendo uso de
medicamentos são originários dos países europeus, que detêm tradição nos estudos de utilização de medicamentos desde o nascimento dessa modalidade de investigação no final da década de $60^{(4)}$.

Considerando as constantes mudanças no mercado farmacêutico que influenciam 0 padrão de prescrição e até mesmo o de automedicação, o perfil de uso de 
Ribeiro, N.K.R.; Leite, L.L.B.; Pontes, Z.B.V.S. Revista Eletrônica de Farmácia Vol. X (1), $16-26,2013$.

medicamentos na gravidez se faz urgente e necessário ${ }^{(2)}$.

Tendo em vista, o grande consumo de medicamentos como ferramenta farmacológica, muitos trabalhos vêm sendo realizados almejando disponibilizar indicadores de consumo durante a gestação, elucidando os riscos que a utilização dos medicamentos podem trazer para seus usuários.

Com o objetivo de contribuir com o uso racional dos medicamentos em gestantes buscou-se identificar os medicamentos utilizados por mulheres durante o pré-natal de um hospital público de ensino, no município de João Pessoa-PB.

\section{MATERIAL E MÉTODOS}

Trata-se de um estudo transversal realizado com 90 gestantes, em idades gestacionais variadas, cuja média de idade foi 24 anos, sendo que $25,6 \%$ das gestantes tinham idade superior a 30 anos e $20,0 \%$ entre 15 e 20 anos. Os valores encontrados para a idade gestacional foram: $2,2 \%$ com menos de 4 semanas de gestação, 2,2\% com 5 a 7 semanas de gestação; 5,6\% com 8 a 10 semanas; 24,4\% com 11 a 13 semanas e, 65,5\% com mais de 13 semanas. A classificação por idade gestacional foi baseada nos períodos em que feto está mais responsivo a interferências externas como medicamentos.

Os medicamentos foram classificados segundo o sistema de Classificação Anatômico Terapêutico Químico (ATC) da Organização Mundial de Saúde e quanto ao risco de uso na gravidez de acordo com o Food and Drug Administration (FDA). Essa classificação enquadra os medicamentos em cinco categorias:

- Categoria A: medicamentos para os quais não foram constatados riscos para o feto em ensaios clínicos cientificamente desenhados e controlados;

- Categoria B: medicamentos para os quais os estudos com animais de laboratório não demonstraram risco fetal (mas não existem estudos adequados em humanos) e medicamentos cujos estudos com animais indicaram algum risco, mas que não foram comprovados em humanos em estudos devidamente controlados;

- Categoria C: medicamentos para os quais os estudos em animais de laboratório revelaram efeitos adversos ao feto, mas não existem estudos adequados em humanos e medicamentos para os quais não existem estudos disponíveis;

- Categoria D: medicamentos para os quais a experiência de uso durante a gravidez mostrou associação com o aparecimento de 
Ribeiro, N.K.R.; Leite, L.L.B.; Pontes, Z.B.V.S. Revista Eletrônica de Farmácia Vol. X (1), $16-26,2013$.

má-formações, mas que a relação risco-benefício pode ser avaliada;

- Categoria X: medicamentos associados com anormalidades fetais em estudos com animais e em humanos e ou cuja relação riscobenefício contra indica seu uso na gravidez.

A pesquisa foi realizada no serviço de pré-natal do Hospital Universitário Lauro Wanderley (HULW) no município de João Pessoa, PB. O serviço de assistência pré-natal do HULW funciona de segunda a sexta-feira, no turno da manhã e da tarde, atendendo semanalmente uma média de 100 gestantes.

Foram realizadas entrevistas semi-estruturadas, a partir de um questionário que continha oito perguntas relacionadas à identificação da paciente, idade, tempo de gravidez, utilização de medicamentos no mês anterior da pesquisa, quais medicamentos utilizados e se foram prescritos ou não, objetivando identificar o perfil de utilização de medicamentos pelas gestantes no período pré-natal.

A coleta de dados ocorreu durante os meses de outubro e novembro de 2010, com carga-horária dedicada a pesquisa de campo no HULW equivalente a 12 horas semanais.

O projeto foi aprovado pelo comitê de ética em pesquisa (CEP) do mesmo hospital e todos os entrevistados assinaram o termo de consentimento livre e esclarecido permitindo a coleta dos dados.

\section{RESULTADOS}

Das 90 mulheres gestantes apenas 4,44\% não fizeram uso de quaisquer medicamentos durante a gestação até o momento da pesquisa, e o total de medicamentos utilizados pelas gestantes foi de 203 medicamentos. A média de medicamentos utilizados foi de 2,3 por gestante. E cerca de $8 \%$ das entrevistadas apresentavam-se no primeiro trimestre da gestação.

De acordo com a classificação Anatomical Therapeutic Chemical Classification System da OMS (ATC), podemos afirmar que os medicamentos mais utilizados pelas gestantes foram: o sulfato ferroso (B03 preparados antianêmicos) 31,03\%; paracetamol (N02 analgésicos) 4,93\%; brometo de escopolamina (A03 medicamentos para disfunções funcionais gastrointestinais) 4,93\%; Dimenidrinato (A04 antieméticos) 2,46\%; metildopa (CO2 antihipertensivos) 1,97\%; dipirona (M01antiinflamatórios) 1,45\%, e nistatina (J02 antimicótico) 0,99\%. (antiinflamatórios) 1,45\%, e nistatina (antiinfeccioso) 0,99\%. 
Ribeiro, N.K.R.; Leite, L.L.B.; Pontes, Z.B.V.S. Revista Eletrônica de Farmácia Vol. X (1), $16-26,2013$.

De acordo com o segundo nível da classificação ATC obteve-se o seguinte resultado: 62,07\% antianêmicos, 5,42\% antiinfeccioso, $4,93 \%$ medicamentos para disfunções gastrointestinais, 4,53\% analgésico, $4,43 \%$ antieméticos, 3,45\% antihipertensivo, e $2,46 \%$ antiinflamatório. Conforme estão ilustrados no gráfico 1.

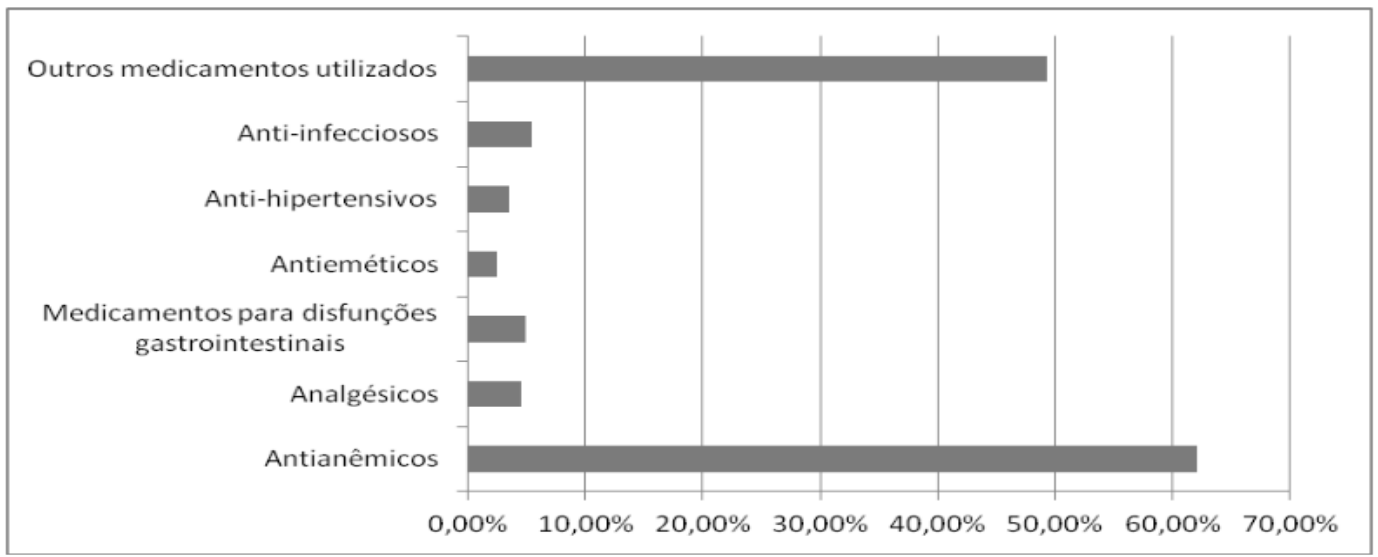

Gráfico 1: Distribuição dos medicamentos mais utilizados pelas gestantes de acordo com o segundo nível da classificação ATC (Anatomical Therapeutic Chemical Classification System).

O nifedipino e propranolol representaram o menor percentual (0,99\% quando somados) encontrado entre as especialidades de antihipertensivos relatadas pelas gestantes. Cerca de $11 \%$ das entrevistadas afirmaram desconhecer o medicamento em uso e/ou sua indicação, nesse caso o medicamento não foi contabilizado ou foi registrado em uma classe terapêutica de acordo com sua indicação. Além disso, percentagem de automedicação na população estudada foi de $5,42 \%$.

Para uma observação mais criteriosa dos possíveis efeitos tóxico/teratogênico, os medicamentos relatados pelas gestantes foram distribuídos na classificação FDA (Food and Drug Administration), tendo como resultado: $18,75 \%$ na classe $A, 43,38 \%$ na $B, 31,25 \%$ na $C$, $0 \%$ na $D$ e $X$ e $6,62 \%$ não identificado, como mostra o gráfico 2 . 
Ribeiro, N.K.R.; Leite, L.L.B.; Pontes, Z.B.V.S. Revista Eletrônica de Farmácia Vol. X (1), $16-26,2013$.

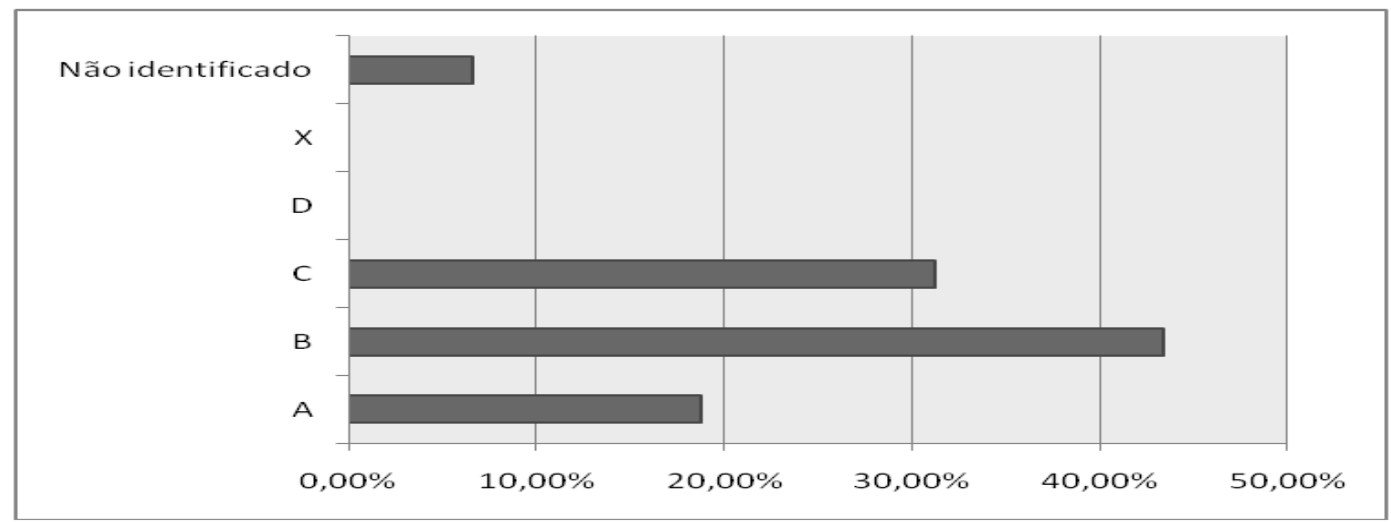

Gráfico 2: Distribuição dos medicamentos utilizados pelas gestantes de acordo com classificação de risco do FDA (Food and Drug Administration).

O uso de suplementos alimentares, sobretudo ácido fólico e sulfato ferroso, representaram $66,0 \%$ dos medicamentos usados pelas gestantes atendidas no HULW.

\section{DISCUSSÃO}

A porcentagem de medicamentos utilizados pelas gestantes nesta pesquisa foi condizente com os $6,14 \%$ relatado pela Norwegian Prescription Database - NorPD(5). A média de utilização de medicamentos por pessoa ficou próxima a média de 2,4 encontrada em estudo na cidade de Natal- RN. ${ }^{\left({ }^{6}\right)}$

O consumo de medicamentos durante a gestação, sobretudo no primeiro trimestre, exige perícia e prudência do usuário, uma vez que o período da embriogênese é a faixa temporal crítica de susceptibilidade do feto aos agentes teratogênicos.

No Brasil, constatou-se que a média de medicamentos utilizados por gestantes variou entre 3,63 e 4,2 $2^{(7-8)}$. Esse número é apenas um valor relativo, que não necessariamente irá condizer com os valores absolutos e reais do uso de medicamentos na gestação, pois, os dados obtidos em pesquisas são pertinentes apenas ao uso da medicação prescrita, e quando a automedicação é relatada, os dados colhidos levam em consideração apenas as memórias da gestante, sendo assim, propício a erros.

Em relação aos medicamentos mais utilizados pelas gestantes, Gomes (1999), aponta: vitaminas e sais minerais $(86,7 \%)$, vacinas $(39,7 \%)$ analgésicos (30,9\%), antiácidos $(27,0 \%)$, antiespasmódicos $(25,1 \%)$, cremes ou pomadas de uso tópico vaginal $(24,0 \%)$ - referindo-se 
Ribeiro, N.K.R.; Leite, L.L.B.; Pontes, Z.B.V.S. Revista Eletrônica de Farmácia Vol. X (1), $16-26,2013$.

à via e não à classe - e antibióticos $(15,3 \%)$ encontram-se entre os mais utilizados.

No tocante aos antihipertensivos há descrições na literatura dos riscos de malformações congênitas e eventos perinatais associados ao uso do nifedipino e do propranolol. Os betas bloqueadores podem levar a um quadro de hipoglicemia devido ao aumento dos níveis de insulina e diminuição dos níveis de glucagon, já os bloqueadores de canais de cálcio levam a hipocalcemia, que por sua vez, é uma conhecida causa de convulsões e mortalidade em neonatos ${ }^{(9)}$.

Destacando a falta de conhecimento da terapêutica, por parte da população estudada, o autor Oenniing(10), ressalta que a ausência de informações sobre a terapêutica pode trazer várias consequências como: a não adesão ao tratamento, o insucesso terapêutico; retardo na administração do medicamento, agravando o quadro clínico do paciente; aumento da incidência de efeitos adversos, por inadequado esquema de administração e/ou duração do tratamento; dificuldades na diferenciação entre manifestações da doença e efeitos adversos da terapêutica; e incentivo à automedicação, bem como outras sérias consequências, que podem piorar o estado de saúde do paciente.
Sobre o valor de automedicação encontrada na pesquisa foi menor, quando comparada aos valores encontrados em estudo no município de Bandeirantes - Paraná, com 8,2 e na cidade de São Paulo, com prevalência de automedicação de $33,5 \%{ }^{(11-8)}$.

No que diz a classificação dos medicamentos, em relação aos riscos na gravidez, em estudo nos Estados Unidos apontou que $5,8 \%$ das gestantes fez uso de medicamentos pertencentes à categoria $\mathrm{D}$ ou $\mathrm{X}$ da classificação da FDA ${ }^{(12)}$.Tais resultados divergem do valor obtido em nosso estudo já que não foi encontrado nenhum medicamento referente a esta classe, apesar de que cerca de $75,0 \%$ dos medicamentos utilizados pertencerem às classes $B$ e $C$ que não há estudos adequados em humanos que comprovem sua segurança.

A propósito do consumo de suplemento alimentar na gestação os valores obtidos demonstra consonância, pois, estudo realizado em Camden, Nova Jersey que afirma que 0 uso de suplementos polivitaminicos/poliminerais pode dimunuir os riscos associados à nutrição para a mãe e para o feto, além de reduzir os riscos de parto prematuro e de baixo peso ao nascer à metade ${ }^{(13)}$.

O fato da alta taxa de nascimentos prematuros, que muitas vezes dá lugar ao baixíssimo peso ao 
Ribeiro, N.K.R.; Leite, L.L.B.; Pontes, Z.B.V.S. Revista Eletrônica de Farmácia Vol. X (1), $16-26,2013$.

nascer, é a maior causa de sobre o perfil de utilização de mortalidade infantil nos Estados medicamentos em gestantes, visto Unidos e de morbidade e incapacidade entre as crianças que sobrevivem ${ }^{(14)}$, surgindo assim a relevância do uso de suplementos alimentares, sobretudo, no que se refere ao primeiro trimestre da gestação.

Os resultados apresentados demonstraram semelhanças com os da literatura, mas também reforça a necessidade de mais conhecimento que a maioria pode trazer riscos à mãe e ao feto.

É a partir da determinação desse perfil que medidas educacionais podem promover à promoção do uso racional de medicamentos entre as grávidas para garantir o uso correto e seguro dos medicamentos na gravidez.

\section{REFERÊNCIAS}

1. Pereira VOM, Acurcio FA, Júnior AAG, Silva GD, Cherchiglia ML. Perfil de utilização de medicamentos por indivíduos com hipertensão arterial e diabetes mellitus em municípios da Rede Farmácia de Minas. Cad. Saúde Pública. $2012 ; 28(8): 1546-58$.

2. Fonseca MRCC, Fonseca E, Bergsten-Mendes G. Prevalência do uso de medicamentos na gravidez: uma abordagem farmacoepidemiológica. Rev. Saúde Pública. 2002;36(2):205-12..

3. Silva $A L$, Ribeiro $A Q$, Klein $C H$, Acurcio $F A$. Utilização de medicamentos por idosos brasileiros, de acordo com a faixa etária: um inquérito postal. Cad. Saúde Pública. 2012;28(6):1033-45

4. Osorio-de-castro CGS, Luiza VL, Pepe VLE, Cosendey MAE, Miranda FF, Bermudez JAZ et al. Uso indicado e uso referido de medicamentos durante a gravidez. Cad. Saúde Pública. 2004; 20 Sup 1:S73-82.

5. Espnes MG, Bjorge T, Engeland A. Comparison of recorded medication use in the Medical Birth Registry of Norway with prescribed medicines registered in the Norwegian Prescription Database. Pharmacoepidemiol Drug Saf. $2011 ;(20): 243-8$. 
Ribeiro, N.K.R.; Leite, L.L.B.; Pontes, Z.B.V.S. Revista Eletrônica de Farmácia Vol. X (1), $16-26,2013$.

6. Guerra GCB, SILVA AQB, França LB, Assunção PMC, Cabral RX. Utilização de medicamentos durante a gravidez na cidade de Natal. Rev Bras de Ginecol Obstet. 2007;30(1):12-8.

7. Maeda ST, Secoli SR. Utilização e custo de medicamentos em gestantes de baixo-risco. Rev Latinoam Enferm. 2008;16(2):266-71.

8. Gomes KRO, Moron AF, Silva RS, Siqueira AAF. Prevalência do uso de medicamentos na gravidez e relações com as características maternas. Rev. Saúde Pública. 1999;(33):246-54.

9. Davis RL, Eastman D, Mcphillips H, Raebel MA, Andrade SE, Smith D et al. Risks of congenital malformations and perinatal events among infants exposed to calcium channel and beta-blockers during pregnancy. Pharmacoepidemiol Drug Saf. 2011;(20):138-45.

10. Oenniing D, Oliveira BV, Blatt CR. Conhecimento dos pacientes sobre os medicamentos prescritos após consulta médica e dispensação. Rev C S Col. 2011;16(7):3277-83.

11. Melo SCCS, Pelloso SM, Carvalho MDB, Oliveira NLB. Uso de medicamentos por gestantes usuárias do Sistema Único de Saúde. Acta Paul Enferm. 2009;22(1):66-70.

12. Andrade SE, Raebel MA, Morse AN, Davis RL, Chan KA, Finkelstein JA et al. Use of prescription medications with a potential for fetal harm among pregnant women. Pharmacoepidemiol Drug Saf. 2006;15:546-54.

13. Scholl TO, Hediger ML, Bendich A, Schall JI, Smith WK, Krueger PM. Use of multivitamin/mineral prenatal supplements: influence on the outcome of pregnancy. Am J Epidemiol. 1997;46:134-41.

14. Wilcox A, Skjaerven R, Buekens P, Kiely J. Birth weight and perinatal mortality: a comparison of the United States and Norway. JAMA. 1995;273(9):70911. 